\title{
La ecología-mundo bajo plástico: un análisis de la articulación entre la explotación de la naturaleza, el racismo y el sexismo en la producción de frutos rojos de Huelva
}

\author{
Emmanuelle Hellio \\ y Juana Moreno Nieto*
}

\begin{abstract}
RESUMEN
Este artículo aborda los vínculos existentes entre la explotación de la mano de obra migrante y el desarrollo de la agricultura intensiva en la provincia de Huelva, España. El objetivo es describir cómo la apropiación explotación de la naturaleza humana y extrahumana (Moore, 2015) convergen en el cultivo de la fresa.Tomamos como punto de partida la teoría del capitalismo como ecología-mundo de Moore y la articulamos con las aportaciones del feminismo materialista y los análisis de la economía política sobre el continuum de control de la movilidad del trabajo en la historia del capitalismo. A partir de este posicionamiento teórico, analizamos el modo en que este cultivo extrae su rentabilidad de la asignación a circular de temporeras marroquíes empleadas a través del programa de migración temporal conocido como contratación en origen. Este programa permite al sector disponer de una mano de obra poco costosa y flexible que garantice la rentabilidad del cultivo, y que retorna a su país de origen al final de la temporada.Veremos que para ello se ha dirigido exclusivamente a mujeres con hijos pequeños a su cargo. La circulación entre país de origen y de trabajo de las jornaleras marroquíes y su adecuación a las exigencias del sector se fundan en las asimetrías de género, clase y etnia existentes. Concretamente, el programa implementa una doble dependencia de los hogares de trabajadoras hacia los ingresos de la temporada, por un lado, y hacia su participación en la economía doméstica, cuando regresan, por otro. Leemos esta subordinación de la reproducción por la producción como una apropiación de la naturaleza tal y como la define, de manera amplia, Moore.
\end{abstract}

El artículo está basado en el material etnográfico recogido entre 2009 y 2012, y actualizado en 2019, en los lugares de vida y trabajo de las temporeras. Su estructura es la siguiente. En una primera parte se exponen las principales características de la producción de frutos rojos en Huelva. En un segundo momento, se describe cómo la contratación de temporeras marroquíes ha sido una estrategia del modelo productivo que había agotado los recursos presentes en el territorio. En la tercera parte, se abordan las consecuencias que el modelo de contratación en origen tiene sobre las condiciones de vida y trabajo de las temporeras y se describen los límites del programa que se está viendo cuestionado por la emergencia de movilizaciones y resistencias.

\section{Palabras clave}

Contratación en origen; temporeros marroquíes; fresa; imbricación clase raza sexo; frontera; ecologíamundo.

\section{TITLE}

World-ecology under plastic: an analysis of the articulation between the exploitation of nature, racism and sexism in the production of berries in Huelva (Spain)

\section{Extended Abstract}

The province of Huelva, in Andalusia (Spain), is the first strawberry exporting area in Europe. Based on an intensive use of inputs applied on sterilized land, this sector is an archetype of agricultural industrialization, marking the decisive influence of

DOI:

https://doi.org//0.15366/relacionesinternacionales202I.47.006

Formato de citación recomendado:

HELLIO, Emmanuelle y MORENO NIETO, Juana (202I)."La ecología-mundo bajo plástico: un análisis de la articulación entre la explotación de la naturaleza, el racismo y el sexismo en la producción de frutos rojos de Huelva", Relaciones Internacionales, $n^{\circ}$ 47, pp. I25-142.
* Emmanuelle HELLIO, Becaria postdoctoral de la Coordinación de Humanidades en el Instituto de Investigaciones sociales de la UNAM. Contacto: manouhellio@ hotmail.com

\section{Juana MORENO} NIETO,

Doctora en

Antropología. Su principal línea de investigación es el cruce entre género, migración y trabajo agrícola. Pertenece al Área de Sociología del Departamento de Economía General de la Universidad de Cádiz. Contacto: juanamorenonieto@ yahoo.es

Recibido:

22.09.2020

Aceptado:

21.04.2021 
capitalist activity on the biosphere right down to the landscape.

Considered red gold for decades, this monoculture entered into crisis in the mid-1990s. Despite the continuous increase in input costs, the price per kilogram of strawberries remains stable, with supermarkets and input suppliers controlling the agrifood chain. To maintain profitability, farmers have followed three strategies: increasing production per hectare through technical intensification of production, introducing new berries cultures, and making wages an adjustment variable. This last one, has been possible through the employment of a diversified migrant workforce. Since the 90 's, various labor substitutions processes have resulted in a segmented labor market by origin, gender, migration status and work relationship. From the year 2000 , an important part of the labor force have been women employed through a temporary migration program named contratación en origen. Morocco has been the main country of recruitment since 2008. Besides Moroccan workers, the sector employs North andWest African workers, with or without work permits, an important number of Eastern European workers coming seasonally through work agencies, and Spanish women workers, mainly in the packing stations. The rise of unemployment caused by the economic crisis limited the contratación en origen between 2012 and 2017 . However, this program is very appreciated by employers who have been demanding its reinstatement, as it guarantees a flexible and non-demanding labor force, available throughout the campaign, even if at certain times the work is scarce. In 2017, it was reactivated with more workers than ever.

Based on qualitative material gathered between 2009 and 2012, and updated in 2019, we will address several dimensions of an agro-migration regime constitutive of this land designed by capitalist world-ecology. The paper will focus on the contratación en origen held with Morocco since 2006. Financed by European Union money, this program aims to facilitate the movement of women workers who must return to Morocco after the season ends. To this end, a series of criteria are established based on racist and sexist stereotypes that define rural Moroccan women mainly as mothers and wives to justify their return to the country when strawberry season is over.To participate in the program, it is necessary to be a woman of rural and poor origin and to have dependent children under 14 years old. It means that capital accumulation in this agriculture is based on control over female farmworkers reproduction. Taking up the contributions of materialist feminism and cross-referencing them with political economy and world-ecology theory, we analyze this subordination of reproduction by production as an appropriation of nature as defined by Moore. Capitalist accumulation expands through commodity fronts (20I5). Competition leads to a perpetual search for new territories - spatial or social - that have not yet been commodified and whose conquest opens new cycles of profitability. Capitalism appropriates these borderlands, reclaiming the free labor of women, nature and colonies (Mies, 1986) and justifying it by the fact that these cheap natures are objects that can be appropriated. Naturalization is the ideology that allows the material exploitation and appropriation of the "free gifts of nature".

In this paper, we argue that, in intensive agriculture, accumulation is based on the exploitation of the web of life, embedded in control over the reproduction of female foreign farmworkers. We show how the imbrication between racism and sexism transforms Moroccan women into inputs for intensive agriculture and address the two dimensions of these power relations: their material appropriation and the ideology that identifies women and racialized people as nature that sustains the former.

We analyze how borders implemented by the contratación en origen mobilize gender and race to allow workforce exploitation. This temporary migration program is based on an economic articulation and a physical separation of the time-spaces of production and reproduction of labor power. Moroccan farmworkers are recruited as appropriated women (Guillaumin, 1992). It is because they carry out the bulk of domestic work, because they are materially involved in the rearing of children, and because this activity is considered their legitimate and main activity, that they are seen as ideal seasonal workers. That is to say, the women will work hard for their children that remain on the other side of the border and they will return home at the end of the season.

The constraints implied by the process of recruitment are reinforced by a legal captivity induced by the fact that residence permits are linked to a specific employer. In addition to this juridical captivity, the confinement in the dwellings weakens these workers' capacity to negotiate their working conditions. The farms are often far from the villages. Isolation is increased by the fact that farmworkers generally do not speak Spanish and do not have any other means of transportation than the one normally provided once a week by the employer for shopping. The mechanisms of control over women's bodies and sexuality are furthermore mobilized to impose discipline and control over workers, as well as to avoid them leaving the Program. The stigma of the woman of bad life and the prostitute, attributed to those who go out at night or to those who leave the Program to stay irregularly in Spain, patrols the borders of temporary work. This system constructs a vulnerable labor force ready to accept the poor working and living conditions offered. However, as Burawoy (1975) pointed out, the interdependence between home and host countries and the separation of production and reproduction tend to erode over time, usually leading to resistance and eventual labor replacement. In 2018 the pact of silence regarding the living and working conditions of foreign seasonal workers was finally broken. The collective mobilizations of Moroccan seasonal workers were organized to denounce poor living and working conditions and the existence of sexual abuse. This questioned the core of a recruitment program that had been designed as an example of ethical and orderly migration for 20 years. At the end of the season, various actors also reported that a significant number of female workers had not returned to Morocco at the end of their contracts. We read these forms of collective and individual resistance as signs of the erosion of this program. We will have to wait to see the impact of these emerging acts of resistance.

\section{KEYWORDS}

Hiring at source; Moroccan farmworkers; strawberry; intersectionality class race sex; border;World-ecology. 
La provincia de Huelva, en Andalucía, alberga desde hace cincuenta años la principal zona exportadora de fresas de Europa. Se trata de un sector arquetípico de agricultura industrial. Basado en el uso intensivo de insumos importados que se aplican sobre una tierra previamente esterilizada, esta agricultura ha transformado profundamente el paisaje local, mostrando la influencia decisiva de la actividad capitalista sobre la biosfera. Basta con poner un pie en el mar de plástico constituido por 10.000 hectáreas de invernaderos para percibir que nos encontramos ante un territorio cuyos recursos están agotados, un frente pionero del cual queda poco por extraer (Moore, 20I7).

En un contexto en el que la tasa de beneficios del cultivo ha ido descendiendo en las últimas décadas, los agricultores han seguido tres estrategias para mantener la rentabilidad del sector. La primera, ha consistido en la intensificación de la producción a fin de aumentar el rendimiento por hectárea, lo que no ha hecho sino profundizar la dependencia del cultivo hacia las grandes corporaciones de insumos. La segunda estrategia ha consistido en la diversificación de los cultivos a través de la introducción de otros frutos rojos como el arándano o la frambuesa. Finalmente, la tercera estrategia se ha traducido en la búsqueda de recursos humanos en el exterior del territorio para abaratar costes y evitar el agotamiento del modelo, ya sea a través de la deslocalización hacia el sur o el empleo de mano de obra migrante.

En este artículo mostraremos cómo el régimen agromigratorio de la contratación en origen, desarrollado en Huelva desde el año 2000, ha llevado a importar madres marroquíes para exportar fresas. A partir de material etnográfico recogido entre 2009 y 2012, y actualizado en 2019, trataremos de comprender los vínculos existentes entre la explotación de la mano de obra migrante y el desarrollo de la variante mediterránea del modelo de producción agrícola californiano. El objetivo es describir cómo la apropiación explotación de la naturaleza humana y extrahumana (Moore, 20I5) convergen en el cultivo de la fresa.

Para ello, partiremos de la teoría del capitalismo como ecología-mundo (Moore, 20I5) y la articularemos con los aportes del feminismo materialista y los trabajos sobre la movilización del trabajo racializado por parte de los países centrales del capitalismo avanzado, en concreto, para el sector agrícola.

Para Moore (20I5), la acumulación capitalista se expande a través de fronteras de producción'. La competencia lleva a una búsqueda constante de nuevos territorios -espaciales o sociales- que aún no hayan sido mercantilizados y garanticen la acumulación. El capitalismo se apropia de estas zonas fronterizas, recuperando el trabajo gratuito de las mujeres, la naturaleza y las colonias (Mies, 1986). Estos espacios forman un frente de mercancías cuya conquista abre nuevos ciclos de rentabilidad, ligados a la explotación de los valores de uso de las "cuatro naturalezas baratas" identificadas por Moore. Estas naturalezas —la fuerza de trabajo, la energía, los alimentos

Aquí es interesante pensar en la etimología de la palabra frontera que nos acerca a un mejor entendimiento de su función para el capitalismo. Moore nos recuerda que las fronteras son también frentes de apropiación colonial. Un frente está más diseñado para avanzar que para retener. A contracorriente del discurso actual sobre las fronteras, recuperamos la idea de que la frontera jurídico política creada por el dispositivo de contratación en origen, y que transforma mujeres en mano de obra barata, es un dispositivo de conquista y no de protección. Esta conquista se opera a través de leyes migratorias dirigidas al control de la movilidad, lo que rompe con el consenso según el cual el capitalismo es un sistema basado en la libre circulación. 
y las materias primas - son baratas porque están ideológicamente devaluadas (Moore, 2017). Existe, por tanto, un nexo sistémico entre el trabajo femenino y racializado no remunerado y el desarrollo capitalista. La contribución de la ecología-mundo es que sienta las bases para una potencial convergencia de las luchas ecologistas, feministas y antirracistas contra el capitalismo. A diferencia de la tradición marxista, reconoce que la acumulación no sólo está vinculada a los procesos de explotación del trabajo, sino también, y sobre todo, a la apropiación barata o gratuita de actividades vitales (trabajo humano no remunerado y actividades extrahumanas). Para que estas actividades sean apropiables, deben ser construidas como objetos exógenos a la sociedad, lo que para la fuerza de trabajo femenina y racializada implica, como veremos a continuación, su naturalización.

En este marco, las mujeres y los inmigrantes representan una frontera - social- del capitalismo (Moore, 2017), constituida por las relaciones de género y de raza. El feminismo materialista y los trabajos de economía política sobre la inmigración laboral lo han demostrado al señalar cómo la idea de la naturaleza, construida como un recurso externo explotable, se extiende también a las mujeres y a las personas racializadas, legitimando su apropiación ${ }^{2}$ (Guillaumin, 1992). La apropiación en estos dos casos ha sido históricamente promovida por el estado a través de una construcción legal de estas personas como menores, extranjeras, esclavas, no ciudadanas, etcétera. Ello ha llevado aparejado una privación total o parcial de la libertad de movimiento, de trabajar o de tener descendencia para los esclavos, los colonizados y las mujeres y ha tenido un impacto directo en su participación en el mercado de trabajo (Moulier Boutang, 1998, p. 537). En la actualidad, esta labor de precarización legal se lleva a cabo a través de las políticas migratorias y la asignación de un estatus subalterno a los migrantes y personas extranjeras. A través de los regímenes migratorios, los estados desempeñan un papel clave en la construcción y canalización de una mano de obra flexible, procedente de la periferia, que responde a las condiciones y tiempos requeridos por la producción. Esta mano de obra es excluida por mecanismos legales que pretenden reducir el trabajador o trabajadora al trabajo (Sayad, I99I) e impedir su asentamiento en los polos productivos (Molinero y Avallone, 2016).

Hoy en día, en los enclaves agrícolas globalizados, la frontera, al igual que las relaciones de raza y género que reconfigura y actualiza, es productiva. Esta compleja construcción se apoya en las relaciones asimétricas existentes entre el Norte y el Sur Global. Así, podemos afirmar que la frontera es tan social como espacial y se reactiva dentro de los propios estados, creando márgenes en el espacio central (Walker y Moore, 2018) a través de las diferencias de estatus legal, las barreras administrativas y otro tipo de exclusiones sociopolíticas.

En el caso de la agricultura intensiva, donde la necesidad de mano de obra es estacional, la explotación de nuevos frentes va acompañada del deseo de preservar parte de la dinámica reproductiva en los espacios a los que retornan los trabajadores y las trabajadoras al final de la campaña. La expansión del capitalismo adopta aquí la forma de una articulación con las economías domésticas pues, para mantenerse en el tiempo, requiere no destruir totalmente las bases de la reproducción. Sin embargo, esta articulación, que constituye el corazón de cualquier sistema de

Para Guillaumin, la apropiación permanente de la clase de las mujeres por parte de la clase de los hombres se asemeja a la esclavitud de las plantaciones. Esta dominación con carácter permanente implica la apropiación del tiempo, los productos del cuerpo, la obligación sexual, la carga física de los miembros dependientes del grupo y la de los hombres y su principal operador es el matrimonio. 
trabajo migrante, tiende a erosionarse con el tiempo, lo que lleva a sucesivas sustituciones de mano de obra, como ilustra muy bien la historia del modelo agrícola californiano (Burawoy, 1976).

Entendemos esta subordinación de la reproducción por la producción como una manera de poner a trabajar la naturaleza tal y como la define Moore. La biopolítica del trabajo temporal (Becerril, 2007), sobre la que nos centraremos en este texto, es sólo una de las formas de expresión de esta subordinación. Esta se expresa igualmente a través de la apropiación de la reproducción de las plántulas de fresa patentadas o en el control de los fenómenos atmosféricos que persiguen los invernaderos. La teoría de la ecología-mundo nos permite reflexionar de manera conjunta sobre la explotación de los recursos ambientales y humanos en el marco de la agricultura intensiva.Al mismo tiempo, a través del análisis del programa de contratación temporal de trabajadoras marroquíes, analizaremos de manera detallada los mecanismos que permiten la explotación de una de las cuatro naturalezas baratas identificadas por Moore, la mano de obra. Nuestro objetivo es tanto reflexionar sobre el paralelismo existente entre la explotación de ambos recursos o naturalezas, como identificar las prácticas sociales que subyacen a cada tipo de relación de explotación. Señalan Walker y Moore que "el capitalismo es una forma de poner a trabajar naturalezas de todo tipo" (Walker y Moore, 2018, p. 50). En este sentido, las zonas de agricultura intensiva en Andalucía se erigen como los espacios naturales globalizados de la ecología-mundo capitalista. Estos espacios - modelados por la acción del estado y los regímenes migratorios existentes - generan nuevos paisajes en los que los árboles han sido sustituidos por túneles de plástico. En ellos crecen el racismo y el sexismo que se imbrican para producir una mano de obra cautiva, fragmentada y desechable que ha evitado, hasta ahora, el agotamiento del modelo agrícola. En este espacio, como muy bien dicen los temporeros y temporeras extranjeras, no queda más energía aprovechable que la que traen ellos consigo.

El artículo está organizado de la siguiente manera. Tras presentar la metodología utilizada, en una primera parte se exponen las principales características de la producción de frutos rojos en Huelva.Argumentaremos que, en este modelo productivo, la mano de obra importada constituye un insumo más, junto a los productos fitosanitarios o el plástico.Al abordar de manera conjunta la explotación de los recursos naturales y de la mano de obra como dimensiones interrelacionadas de un régimen agromigratorio (Gertel y Sippel, 2014) mostraremos los procesos, prácticas y relaciones sociales que configuran a nivel local la ecología-mundo capitalista.

En un segundo momento, detallaremos cómo la contratación de temporeras marroquíes ha sido una estrategia de un modelo de producción extractivista que había agotado los recursos presentes en el territorio. Ante el descenso de la rentabilidad, la estrategia de deslocalización in situ (Terray, 1999) ha mantenido a flote el sector y se ha visto caracterizada por sucesivas sustituciones de mano de obra. Tras acabar con la fertilidad del suelo $y$ ante el aumento de las resistencias o el abandono de parte de la mano de obra local, familiar e inmigrante, este se ha provisto de una nueva reserva de mano de obra construida como ilimitada, natural y barata, las trabajadoras marroquíes contratadas en origen, lo que ha permitido asegurar el crecimiento y gestionar las contradicciones ecológicas internas de este modelo productivo. Veremos cómo el género constituye una dimensión esencial para garantizar la circulación de estas trabajadoras y su adecuación a las exigencias de los empleadores. En la tercera parte, abordaremos las consecuencias que el modelo de contratación en origen tiene sobre las condiciones de vida y trabajo de las 
temporeras y describiremos las contradicciones del programa que, después de veinte años, se está viendo cuestionado por la emergencia de movilizaciones y resistencias.

\section{Metodología}

Este artículo se basa en los resultados de varias investigaciones cualitativas llevadas a cabo en la zona de producción de fresas y otros frutos rojos de Huelva entre 2008 y 2019 . En concreto, una tesina y una tesis doctoral realizadas por sendas autoras entre 2008 y 2012 y una investigación complementaria colectiva en 2019. Durante las distintas fases del trabajo de campo se realizaron más de cien entrevistas en profundidad con los diferentes actores sociales relacionados con el sector (empresarios, instituciones públicas españolas y marroquíes, sindicatos, las ONG...) y, sobre todo, con las temporeras marroquíes contratadas en origen. Las entrevistas tuvieron lugar en Huelva y en los pueblos de origen de las trabajadoras en Marruecos. En casi todos los casos, las entrevistas se realizaron en árabe dialectal con la ayuda de un traductor o traductora nativo. También se realizaron observaciones en los lugares de residencia y trabajo de las jornaleras, así como en los procesos de selección en Marruecos. El carácter intensivo y diacrónico del trabajo de campo ha permitido obtener un conocimiento profundo del sistema de contratación y su evolución, así como de las experiencias migratorias y laborales de las temporeras.

\section{El modelo de producción intensiva de frutos rojos en la provincia de Huelva}

El arco litoral entre la ciudad de Huelva y la desembocadura del Guadalquivir alberga la principal zona de producción de España y Europa que, actualmente, ocupa el sexto lugar en el ranking mundial (Domínguez, 2018). La introducción del cultivo de la fresa en la provincia se remonta a finales de los años cincuenta y reproduce el modelo agrícola californiano, basado en el uso intensivo de tierra, tecnología, capital y mano de obra. Desde el principio se utilizaron variedades americanas y la fruta estuvo destinada a la exportación. Gracias al aprovechamiento de la capa freática del Guadalquivir y a las buenas condiciones geográficas y climáticas, los campos de Huelva pronto se erigieron como un enclave ideal para el cultivo temprano de la fresa.

La propiedad comunal ha jugado un papel importante en la implantación y desarrollo de este monocultivo. Más del $50 \%$ del terreno de la región estaba en manos del estado o de los municipios. La cesión de tierras para el cultivo de fresas se consideró un gesto hacia los jornaleros pobres. Su extensión se vio acelerada por la quema ilegal de pinares, que también eran comunales, y su posterior ocupación. Estas ocupaciones provocaron la desaparición de casi dos mil hectáreas de bosque en cuatro años (Delgado y Aragón, 2006). Por su parte, las administraciones públicas asumieron la financiación y construcción de las infraestructuras necesarias para asegurar el riego, legitimando y promoviendo el desarrollo de esta agricultura. El desarrollo de la fresicultura en Huelva no puede explicarse sin tener en cuenta el papel desempeñado por el estado y las pequeñas explotaciones familiares, pero también la abundancia de jornaleros vinculados a una estructura territorial latifundista en las provincias vecinas. Estos son los factores sociales que, además de las especificidades geográficas y económicas mencionadas, contribuyeron al auge del sector en los años ochenta. 
Por otra parte, cuatro innovaciones principales impulsaron el desarrollo del cultivo durante los años ochenta y noventa: el desarrollo del uso del plástico (invernaderos y plástico negro que cubre el suelo), la innovación biológica (aparición de nuevas variedades patentadas por universidades o corporaciones americanas), la innovación química (desarrollo del uso de fertilizantes y productos fitosanitarios, desinfección del suelo con bromuro de metilo...) y las técnicas de riego por goteo y fertirrigación. Todo ello nos permite afirmar que nos encontramos ante un verdadero sistema de producción agroindustrial.

El carácter intensivo e industrial del sector ha provocado una continua presión sobre los recursos naturales (contaminación de los suelos y agotamiento del acuífero). Asimismo, el sector presenta un alto grado de dependencia económica del exterior debido a que la inversión necesaria para adquirir los insumos es cada vez mayor y a que los precios de venta se mantienen estables debido al control que ejerce la gran distribución. Ello hace que la mayor parte del valor de la cadena agrícola quede fuera del territorio onubense y que la actividad agrícola se sostenga sobre un alto grado de endeudamiento de los agricultores. "La fresa mueve dinero, pero no te da”, señalaba Joaquín, un comerciante de Palos de la Frontera, en 20I I.Ahora bien, cabe decir que, pesar de que la rentabilidad del cultivo disminuye cada año, el sector sigue generando un altísimo volumen de negocio, que alcanzó los 445.000 .000 de euros en 2015 (JDA, 20I7).

Cartografiar la producción de la fresa y otros frutos rojos en Huelva, atendiendo a los espacios que alimentan y son alimentados por el cultivo, implica seguir el trazado de una geografía globalizada en la que el sector participa, en última instancia, de forma marginal. La relación de intercambio desigual aquí descrita se refuerza si tenemos en cuenta los recursos naturales drenados junto con el valor monetario, cuyo agotamiento y contaminación no se contabilizan. En este sistema, la degradación del patrimonio natural, el consumo de agua y tierra y la falta de compensación monetaria por los residuos producidos, quedan relegados al rango de externalidades y no aparecen en los balances monetarios. 


\section{Mar de plástico y fresas en altura}
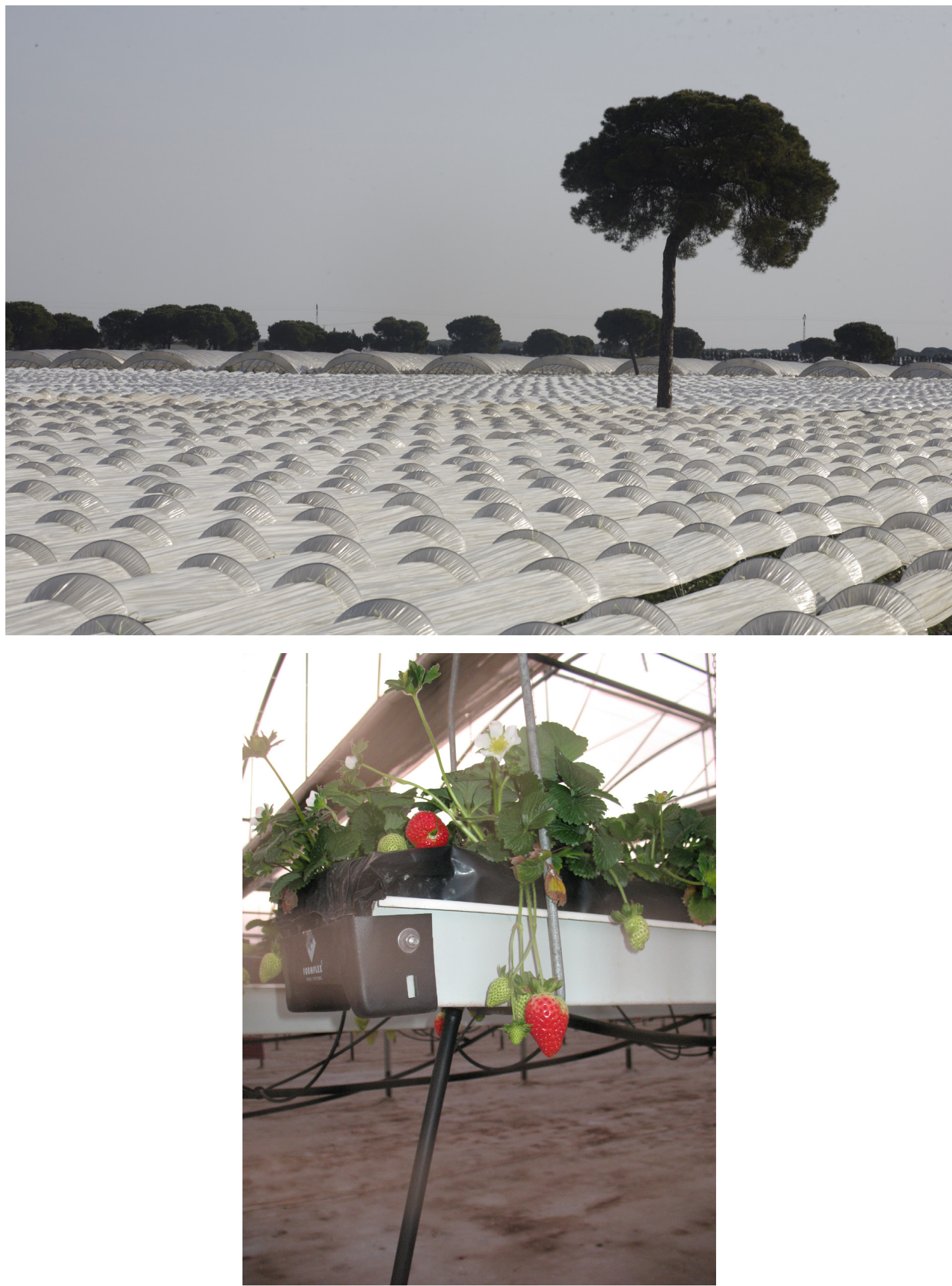

Fuente: suministradas por las autoras. Palos de la Frontera, 2008. 
En efecto, la producción de fresas en Andalucía ha sido, desde el principio un eslabón dependiente de una cadena agroalimentaria globalizada. Esta posición se inscribe en el continuo histórico de explotación de los recursos naturales y de la mano de obra que se inició en la provincia con las minas de cobre en el siglo XIX (Delgado y Aragón, 2006). En los años noventa, el sector enfrentó una importante crisis relacionada con el aumento de la competencia internacional a causa de la aparición de nuevos territorios productores (Marruecos y Egipto, entre otros) y por el hecho de que, a pesar del continuo aumento del coste de los insumos, el precio del kilogramo de fresa se mantenía estable. Para tratar de asegurar el margen de beneficios, los agricultores apostaron por la intensificación del cultivo, a través de un uso intensivo de insumos industriales y la diversificación variedades y frutos. Como señalan Reigada, Moreno y Mozo (202I), ninguna de las estrategias emprendidas por el sector ha pretendido alterar las bases del modelo.

Los elementos modernizadores tienden a proporcionar mecanismos para aumentar los rendimientos, controlar las condiciones de desarrollo de los procesos productivos e implican un alto grado de tecnificación y automatización, así como una mayor dependencia de paquetes tecnológicos, diseñados y elaborados cada vez más lejos del agricultor y de su entorno. El sistema intensivo es la causa de una elevada incidencia de plagas y enfermedades, lo que resulta tanto más preocupante en cuanto que las autoridades empiezan a limitar el uso de determinados productos fitosanitarios. La espiral intensificadora también ha agotado la fertilidad del suelo y ha contaminado las aguas subterráneas. Esta tendencia se ha acentuado en los últimos años con la introducción de nuevas frutas como la frambuesa, la mora o el arándano. En este contexto, sólo aquellas empresas que han invertido en otros segmentos de la cadena o han trasladado su producción más al sur, han conocido un crecimiento sostenido (Hellio y Moreno, 2017).

El mecanismo de endeudamiento continuo, junto a los aleas climáticos y del mercado, coloca en una situación de gran incertidumbre a los productores agrícolas. Por ello, y en un cultivo que demanda grandes cantidades de mano de obra, controlar el factor trabajo adquiere una gran importancia fundamental. Disponer de una fuerza de trabajo fiable, de costos reducidos y que pueda ser utilizada y desechada en función de los requerimientos de la producción y los mercados, se vuelve fundamental en la agricultura globalizada (Berlan, 1987).

En efecto, gran parte del sector intenta superar la incertidumbre controlando las únicas dos variables del proceso de producción sobre las que los productores pueden ejercer cierto poder: la explotación de los recursos naturales y la mano de obra. Estos son dos de los cuatro tipos de naturalezas baratas identificadas por Moore como centrales para la acumulación. Este modelo productivo se basa, por tanto, en diferentes apropiaciones de la naturaleza situadas en distintos eslabones de la cadena. Por un lado, la apropiación de la tierra y el agua comunales y la privatización de la vida a través de la mercantilización de las variedades patentadas. $A$ esta apropiación de la naturaleza, entendida en sentido estricto como medio ambiente, se añade la apropiación de los cuerpos de las mujeres temporeras importadas para la cosecha. Sobre este último aspecto nos detendremos en el siguiente apartado, en el que presentaremos la evolución del sistema de empleo del cultivo y el papel desempeñado dentro del mismo por las trabajadoras marroquíes con contrato en origen. 


\section{El mercado de trabajo en la provincia de Huelva}

\section{I Un sistema de empleo típico de una zona fronteriza}

Como hemos señalado, el mercado laboral de la agricultura onubense ha conocido varios procesos de sustitución de la mano de obra, en los que las políticas migratorias han desempeñado un papel fundamental. Así, en las tres últimas décadas, la presencia de trabajadoras y trabajadores extranjeros, bajo diferentes regímenes migratorios, ha permitido el acceso a una fuerza de trabajo dividida por estatus legal, raza y género. Las trabajadoras marroquíes contratadas en origen forman parte de un complejo mercado de trabajo compuesto por una mayoría de temporeros y temporeras procedentes de Europa del Este (especialmente de Rumanía), jornaleros y, sobre todo, jornaleras españolas, así como trabajadores de África Occidental y del Magreb, residentes en el Estado español.

La contratación de temporeras marroquíes tiene sus orígenes en el año 2000 cuando una gran empresa de Huelva, que había trasladado parte de su producción a Marruecos a finales de los años ochenta, aprovechó la posibilidad abierta por la ley de Extranjería (artículo 42 de la Ley Orgánica 4/2000) para trasladar trabajadoras marroquíes a sus explotaciones españolas. Sin embargo, a nivel sectorial, la mayoría de las cooperativas y empresas utilizaron este canal para contratar a mujeres procedentes del Este de Europa. Habría que esperar a que Polonia, Bulgaria y Rumanía entraran en la Unión Europea para que aumentara el número de contrataciones realizadas en Marruecos. Hay que señalar que la contratación en origen constituyó una respuesta de las instituciones y organizaciones agrarias a un ciclo de movilizaciones protagonizadas, en torno al año 2000, por los jornaleros magrebíes y de África Occidental que trabajaban en el sector (Gualda y Ruíz, 2004). Se decidió, entonces, ordenar las migraciones, de forma que el flujo migratorio se ajustara al máximo a las necesidades de los empresarios.

\section{Evolución de la contratación en origen en la provincia de Huelva 2000-20 I 8}

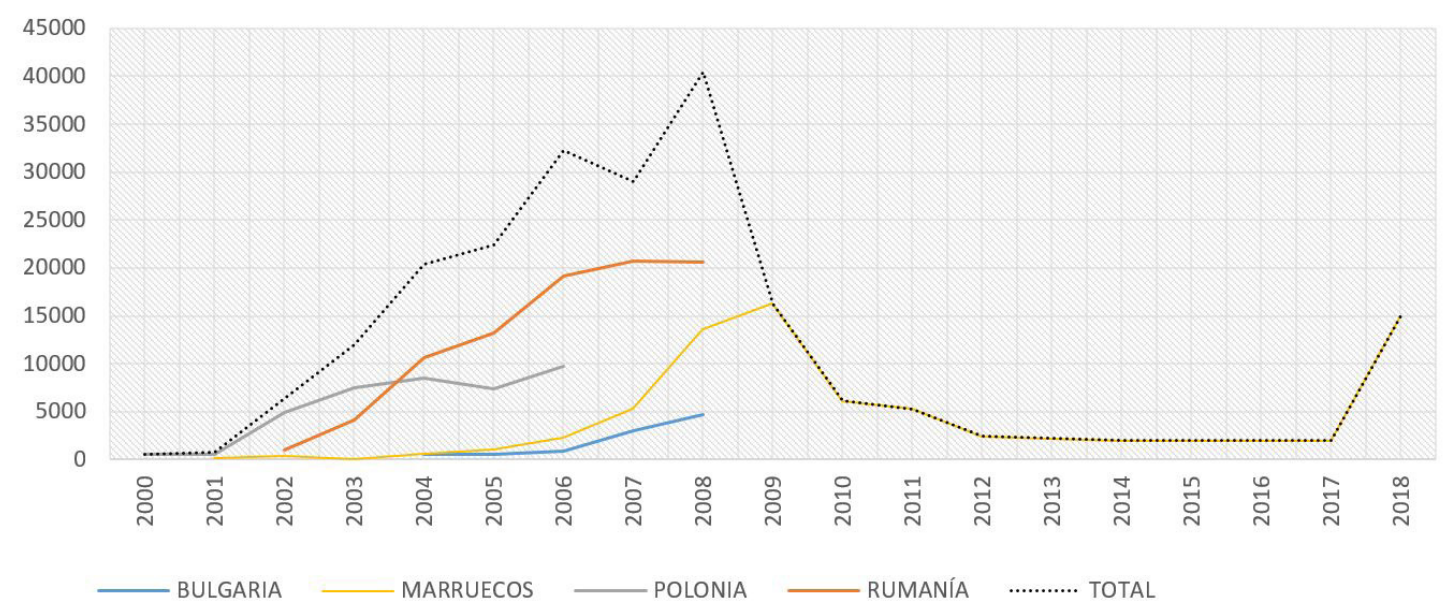

Fuente: De 2000 a 2010, Dependencia de Trabajo y Migración de la Subdelegación del Gobierno. De 201 I a 2018, estimaciones realizadas a partir de anuncios de prensa. Compilación de las autoras. 


\subsection{La contratación en origen en Marruecos: importar madres para exportar fresas}

La contratación de temporeras marroquíes cobra impulso a partir de 2006. En un contexto de promoción y subvención de la migración laboral temporal, el ayuntamiento de uno de los municipios productores de fresas de la provincia obtuvo, en 2005, una subvención de la Comisión Europea de 1,6 millones de euros. Un año después, se firmaría un acuerdo de cooperación con la Agencia Marroquí de Empleo (ANAPEC), que también dispondrá de fondos europeos para poner en práctica programas de emigración laboral a Europa. Aeneas se presenta como un programa de "gestión ética y socialmente responsable de los flujos migratorios laborales África Europa". Con una financiación de más de seis millones de euros en total para organizar la selección, la contratación, el transporte y la mediación entre este nuevo grupo de trabajadoras y los productores y empresarios, el acuerdo Anapec Aeneas Cartaya impulsará la contratación en Marruecos. Así, en 2009 se llegaron a conceder más de 16.000 autorizaciones. No obstante, este sistema de contratación se redujo en gran medida entre los años 2013 y 2017 debido a la elevada tasa de desempleo local derivada de la crisis económica de 2008.

Los contratos en origen están reservados a los nacionales de terceros países, preferentemente de los que hayan firmado un acuerdo con España para regular los flujos migratorios que contenga una cláusula de expulsión. La selección y la firma de los contratos se realizan en el país de origen, que los empresarios eligen en función del peso de la ruralidad, la pobreza y la proximidad geográfica. Los permisos de residencia y de trabajo obtenidos indican la zona geográfica y el sector de actividad autorizados, así como la duración del permiso de trabajo, que corresponde a la duración del contrato y no puede superar los nueve meses. Las trabajadoras contratadas deben regresar a su país al finalizar la temporada para lo que firman un compromiso de retorno antes de partir. La contratación al año siguiente está supeditada a la voluntad del empresario que podrá convocar o no de nuevo a las temporeras, hecho que coloca a estas últimas en una situación de alta dependencia hacia su empleador. Es por todo esto que en la literatura se ha enfatizado la cautividad jurídica que generan este tipo de programas de contratación temporal (Basok, 2009; Moreno, 2009).

En Marruecos, el retorno de las trabajadoras ha sido, desde el principio, un eje estructurante del programa, así como el principal criterio para evaluar su éxito. A fin de reducir la tasa de fugas, las organizaciones agrarias, en connivencia con los responsables del programa Aeneas Cartaya y las autoridades marroquíes, introdujeron criterios de índole familiar que vinieron a sumarse al de sexo, que ya había establecido la contratación exclusiva de mujeres en la experiencia con los países de Europa del Este. Así, a partir de 2006, los contratos se dirigen únicamente a mujeres casadas, divorciadas o viudas, con hijos menores de catorce años a su cargo.También se favorecerá la contratación de trabajadoras procedentes de familias numerosas, con experiencia agrícola y residentes en zonas rurales y pobres. Las estadísticas disponibles sobre la cuota de trabajadoras empleadas entre 2006 y 2010 ofrecen una idea del perfil de las trabajadoras. El contingente estuvo compuesto casi exclusivamente por mujeres (hubo un máximo de ciento ocho hombres por año durante este periodo) con una edad comprendida entre los veinticinco y cuarenta y cinco años (el $7 \%$ tenía entre veinte y veinticinco años y menos del $2 \%$ más de cuarenta y seis) (Hellio, 20l4). Desde el punto de vista del estado civil, más del $50 \%$ de las trabajadoras eran viudas, separadas o divorciadas. La pregunta que se impone irremediablemente es ipor qué los empresarios y 
los gestores han preferido contratar a mujeres, y entre ellas, a las que tienen responsabilidades familiares?

\subsection{La articulación producción reproducción: la biopolítica del trabajo temporal}

Para dar respuesta a la pregunta planteada en el apartado anterior se hace necesario recurrir a un análisis que atienda a la interrelación existente entre el mundo productivo y la esfera de reproducción. De forma holística, la reproducción puede definirse como "el conjunto de actividades y relaciones a través de las cuales se reconstruyen diariamente nuestras vidas y nuestra capacidad de trabajo" (Federici, 20I0, p. 21). Se basa principalmente en el trabajo no remunerado de las mujeres y es el pilar central de la actividad socioeconómica. Esta constatación cuestiona la supuesta independencia del mercado del resto de las estructuras sociales y lleva a asumir que la generación de plusvalía no puede desvincularse de la apropiación del trabajo doméstico y de cuidados que garantiza, entre otras cosas, la reproducción de la fuerza de trabajo (Carrasco, 2006). Así, como señala Kergoat ( 1982 ), la familia y el trabajo doméstico son la piedra angular de la diferenciación del trabajo de hombres y mujeres en el tiempo y el espacio. Por lo tanto, cualquier aproximación al trabajo de las mujeres debe hacerse conjuntamente con un análisis de la situación y el lugar de las mujeres en la esfera de la reproducción.

"El mundo de las trabajadoras forma un sistema en el que el capitalismo y el patriarcado se turnan para explotar dominando y dominar explotando. (...) No existe una yuxtaposición sino un vínculo orgánico entre el trabajo asalariado y el trabajo doméstico" (Kergoat, 1982, p. 69).

Cualquier análisis del trabajo asalariado debe tener en cuenta el trabajo doméstico, pero ¿qué ocurre cuando el trabajo doméstico se desarrolla en un espacio y el trabajo asalariado en otro y estos dos espacios están separados por una frontera?

Para Burawoy (1976), los programas de migración temporal se basan en la construcción de una doble dependencia de la mano de obra del salario estacional y de una economía exterior al enclave productivo que permite garantizar la circulación de los y las migrantes entre las zonas de origen y de trabajo. Es decir, la llegada y retorno de la fuerza de trabajo temporal está asegurada cuando ésta depende de los ingresos de la campaña agrícola, pero sigue participando en la economía doméstica de los hogares cuando regresa al final de la misma. Esta doble dependencia está regulada por el conjunto de disposiciones jurídicas y políticas que conforman la frontera, destinadas a separar los medios monetarios de subsistencia de la mano de obra de los de su reproducción, así como a garantizar la interconexión entre ambos. Esto es, el dispositivo migratorio de contratación temporal permite separar físicamente $y$, al mismo tiempo, articular económicamente, el espacio tiempo de la producción y el de la reproducción de la fuerza de trabajo.

El modelo de contratación en origen lo consigue mediante la articulación generizada entre el empleo temporal y las relaciones de producción doméstica (Delphy, 1970) en las que se insertan las trabajadoras en Marruecos. En un contexto de control de los flujos migratorios, el género contribuye a justificar y naturalizar el mandato de retorno. 
La asignación de las temporeras a circular entre Marruecos y España, impuesta por sus permisos de residencia y trabajo, se consolida apoyándose en las dimensiones materiales e ideológicas de su posición en el hogar. Como lo resume uno de los responsables de Anapec: "el criterio de apego [a los hijos] mejora la tasa de retorno". La feminidad (y más concretamente la identificación de las mujeres como madres) no construye aquí exclusivamente una mano de obra flexible y productiva (Salzinger, 2003), sino también retornable. Estas trabajadoras son, en palabras de Guillaumin (1992), mujeres apropiadas. El hecho de que asuman el grueso del trabajo doméstico y de la crianza de los hijos (Dunezat, 20l6), así como que esta actividad sea considerada como su actividad prioritaria y natural, las convierte en las temporeras ideales, que trabajarán duro para su prole al tiempo que regresarán a casa a ocuparse de ella al final de la temporada ${ }^{3}$. La naturalización de la división sexual de las tareas constituye aquí la justificación ideológica discursiva del sexismo.

\section{Consecuencias sobre la organización del trabajo y el control de la movilidad de las trabajadoras}

¿Cuáles son las consecuencias del sistema de contratación en origen sobre las condiciones de vida y de trabajo de las temporeras en España? Ya en 2006, organizaciones sociales y académicas advertían de las consecuencias del cautiverio legal impuesto por estos contratos sobre las trabajadoras y denunciaban las irregularidades existentes en materia de condiciones laborales y de alojamiento (varios autores, 2006). Veamos en detalle a qué cuestiones se referían entonces, y ahora, estas denuncias.

\section{I. Un régimen de cautividad flexible}

Las condiciones de trabajo en el sector de la fresa y otros frutos rojos se caracterizan por una gran flexibilidad y un ritmo de trabajo intenso. En primer lugar, las trabajadoras no saben cuándo van a empezar o terminar su contrato. Pueden ser llamadas o despedidas de un día para otro ya que, entre otras cosas, no disponen de una copia de su contrato de trabajo. La Dependencia de Trabajo y Migración de la Subdelegación del Gobierno, responsable de la tramitación de estos contratos, recuerda que constituyen un cupo de reserva, que será incorporado en función de las necesidades de la producción y la situación de empleo. Esto resulta especialmente favorable para los empresarios preocupados por asegurarse la presencia de trabajadoras al principio y al final de la temporada, cuando la escasez de horas de trabajo empuja a otros trabajadores comunitarios o residentes a desplazarse hacia otras campañas agrícolas o sectores.

Por otra parte,en el día a día,la constitución de reservas de mano de obra en las explotaciones permite a los productores disponer de suficientes trabajadoras durante los períodos de máxima producción. Ello implica que las trabajadoras pueden verse privadas del día de descanso durante esos periodos, al tiempo que sufrirán una escasez de días de trabajo durante el resto de la temporada. Esta flexibilidad impuesta va en contra de los intereses de las trabajadoras y erosiona la rentabilidad de su proyecto migratorio. Así, los ingresos obtenidos son muy variables y pueden oscilar entre los setecientos y los tres mil euros por temporada.

En el marco de la separación buscada entre producción y reproducción, los embarazos de las temporeras son poco apreciados por los agricultores y fueron, al principio de un programa, objeto de retornos forzados a Marruecos. 
Además, los ritmos de trabajo son extremadamente intensos en el sector. Las trabajadoras denuncian un control exhaustivo de la cantidad y la calidad de las cajas recogidas. Aquellas trabajadoras que no cumplan con los objetivos son sancionadas, lo que, normalmente, se traduce en una privación de trabajo el día o días posteriores. El número de cajas que hay que recoger se define en función de la fluctuación de los pedidos, pero también de los precios del mercado, y el sistema de control garantiza que sólo se ponga a trabajar y se pague a la mano de obra efectivamente rentable, como explica aquí un empresario:

"Ayer, seis mujeres no trabajaron, porque recogieron muy pocas cajas [nos muestra la hoja donde se comprueba el rendimiento individualmente]. Recogió catorce cajas. [Coge una calculadora] catorce cajas son setenta kilos, a 0,65 euros el kilo, son 45,50 euros. Esta mujer gana aquí 39, 10 euros, y me cuesta doce euros de seguridad social, lo que supone $5 \mathrm{I}, 10$ euros. También tengo una persona que riega, y en el camión, cuatro mujeres que empacan. Consumo agua y agroquímicos. Las mujeres que recogen veintidós o veintitrés cajas se ganan el sueldo. Los que hacen menos me hacen perder dinero". Marco, propietario de una finca de veintiséis hectáreas, catorce de mayo de 20I I, Moguer.

Al final de la temporada, en algunas explotaciones se trabaja a destajo lo que incrementa el ritmo de trabajo y fomenta la competitividad entre trabajadoras, ya de por si existente debido a la segmentación de los equipos de trabajo por nacionalidad y estatus legal. Estas cadencias de trabajo, especialmente intensas, hacen imposible que algunas temporeras puedan seguir el ritmo. Por otra parte, las horas extraordinarias raramente son remuneradas al precio establecido y, al no saber leer, y menos aún los documentos escritos en español, las temporeras tienen dificultades para identificar las irregularidades en sus nóminas.

\subsection{El sistema de alojamientos, un paso más hacia el aislamiento y la construcción de la cautividad}

Dado que uno de los pilares fundamentales del programa de migración temporal que nos ocupa es el retorno de las temporeras, no es de extrañar que la movilidad de estas últimas se vea controlada, tanto por los gestores del programa como por los empleadores. Además de la cautividad jurídica que imponen sus permisos de trabajo, vinculados a un empleador concreto, la retención de los pasaportes o de una parte del salario hasta el final de la temporada por parte de algunos empresarios es una práctica que ahonda en la sujeción de las temporeras. Por otra parte, las trabajadoras experimentan un pseudoconfinamiento físico. Suelen residir en las explotaciones agrícolas donde trabajan que se encuentran, por lo general, alejadas de los pueblos, hecho que favorece su aislamiento físico y social. Éstas, además de no conocer el idioma, no disponen normalmente de otro medio de transporte que el que les proporciona el empresario una vez a la semana para hacer compras y gestiones en los pueblos. De ahí que las trabajadoras deban recurrir al autostop o a la red de taxis clandestinos existente en la comarca si desean desplazarse de manera autónoma (Hellio, 20I4). 
Existen, asimismo, controles en las entradas y salidas de las explotaciones, que a menudo están valladas, y las prohibiciones de recibir visitas o realizar salidas nocturnas son habituales ${ }^{4}$. Por otra parte, se anima a las temporeras a utilizar los autobuses fletados por las organizaciones patronales para regresar a Marruecos, lo que complica las posibilidades de abandonar las explotaciones al final de la temporada.

El uso del término fugas para denominar el abandono del programa por una trabajadora resulta ilustrativo de la vocación autoritaria de este tipo de contratación (Duntze, 2008). En concreto, las representaciones de género, por un lado, y la distinción que se hace entre la migración legal, garante de derechos, e ilegal, presentada como intrínsecamente peligrosa, se refuerzan para estigmatizar cualquier movimiento autónomo de las temporeras, eliminando la separación entre el tiempo de trabajo y la vida privada. Así, los empleadores disuaden a las temporeras de establecer relaciones fuera de la explotación, especialmente relaciones sentimentales con varones de origen magrebí, hecho que puede llegar a ser motivo de despido. A éstos, se les acusa de incitar a las temporeras a abandonar las explotaciones bajo engaños y falsas promesas de regularización, cuando no directamente de trata de seres humanos (Zeneidi, 2013). El estigma de la mujer de mala reputación o la prostituta, así como el imaginario del hombre árabe violador, mentiroso y ladrón (Guénif y Macé, 2004), actúan como mecanismos de control de los cuerpos y la sexualidad de las temporeras a fin de garantizar la disciplina y su permanencia en el programa.

\subsection{Resistencias y erosión del programa}

La primavera de 2018 puso fin al pacto de silencio existente sobre las condiciones de vida y estancia de las temporeras extranjeras en la provincia de Huelva. La campaña estuvo marcada por una serie de denuncias públicas sobre los abusos sexuales y laborales sufridos por las trabajadoras contratadas en origen.Además, se calcula que unas dos mil quinientas trabajadoras, es decir, el 17\% del total del contingente, no regresó a Marruecos tras finalizar la temporada.

En un primer momento, la publicación a finales de abril de un reportaje sobre las violaciones y abusos sexuales sufridos por trabajadoras marroquíes del sector puso en el punto de mira internacional el sistema de contrataciones en origen (Müller y Prandi, 2018). Seguidamente, en junio de ese mismo año, las trabajadoras marroquíes de la empresa Doñana 1998, apoyadas por el Sindicato Andaluz de Trabajadores (SAT), intentaron denunciar incumplimientos del contrato y abusos sexuales en la empresa. El empleador organizó el retorno del conjunto de trabajadoras de su finca a Marruecos para evitar que pudieran ratificar sus denuncias. Sin embargo, las trabajadoras, unas cien jornaleras, resistieron y se negaron a montarse en los autobuses. Las redes sociales retransmitieron lo que estaba ocurriendo y los medios de comunicación locales y nacionales se hicieron eco de la situación. A pesar de que la mayoría de las trabajadoras fueron enviadas de vuelta a Marruecos, unos días después, diez de ellas interpondrán denuncias ante los juzgados de lo social de Huelva por vulneración de derechos fundamentales y ante los juzgados de primera instancia e instrucción de La Palma del Condado (Huelva), por delitos contra los derechos de los trabajadores y delitos de trata de seres humanos y lesa humanidad (Echevarría, 2019).

Han tenido que pasar veinte años para que las irregularidades y abusos que permite este programa de migración temporal, erigido como modelo ejemplar de gestión ética y ordenada de

\footnotetext{
Esto complica el acceso de los sindicatos, asociaciones y administraciones españolas y marroquíes a las explotaciones.
} 
las migraciones, hayan traspasado a la opinión pública. Hasta ahora, los intereses económicos del sector, la distancia social entre la población local y las trabajadoras migrantes y el apoyo de las instituciones nacionales y europeas habían contribuido a mantener el statu quo.Y ello a pesar de que se trata de un modelo que se apoya y perpetúa desigualdades de clase, género y nacionalidad. De hecho, las estrategias desplegadas para desacreditar a las denunciantes se inscriben en una retórica racista y sexista por las que se les acusa de ejercer la prostitución o de mentir para obtener la regularización mientras se trasladan las responsabilidades de los abusos a hombres extranjeros (capataces marroquíes o rumanos).

\section{Conclusión}

En este artículo hemos descrito, desde una perspectiva feminista materialista y antirracista, cómo las relaciones de dominación de género y raza, junto a los dispositivos migratorios, permiten a la agricultura onubense extraer sus beneficios de la explotación de las mujeres y la naturaleza. Desde el año 2000, la biopolítica discriminatoria del trabajo temporal (Becerril, 2007) ha sido promovida y subvencionada por las instituciones europeas. El dispositivo jurídico sobre el que reposa el programa de contrataciones en origen y las relaciones desiguales de género han sido utilizados para transformar a las trabajadoras extranjeras en insumos adicionales del modelo productivo. Asistimos así a una sexuación del utilitarismo migratorio, es decir, de la concepción de la migración en términos de costes y beneficios (Sayad, 1986). Diseñado para traer trabajo sin trabajadoras (Morice, 2004), el programa se apoya sobre la asignación prioritaria, material e ideológica, de las mujeres al trabajo de reproducción. Ello permite poner a disposición del sector, de forma segura y flexible, la cuota deseada de braceras en cada momento, mientras se externalizan los costes sociales de este monocultivo.

Asimismo, poniendo en diálogo la teoría de la ecología-mundo con las aportaciones teóricas del feminismo y el análisis del utilitarismo de las políticas migratorias, hemos demostrado el papel central de las fronteras en el acaparamiento de las naturalezas baratas (Moore, 20I5). Contrariamente a las representaciones liberales que nos hablan de un capitalismo sin fronteras y a los discursos militantes que denuncian la multiplicación de muros que impiden toda movilidad, la frontera que el estado pone al servicio del capital aparece más bien como un filtro espacio temporal flexible (Harvey, 1989), generizado y basado en la integración subordinada del Sur al Norte (Berndt y Boeckler, 20l4). Sin embargo, es una herramienta no exenta de contradicciones dado que pretende lo imposible, la separación entre la producción y la reproducción de la fuerza de trabajo. Los programas de migración temporal sólo resultan útiles mientras persiste la ilusión de lo provisorio (Sayad, 1991). Sin embargo, la interdependencia entre el país de origen y el de acogida, y la estricta separación de la producción y la reproducción, tienden a erosionarse con el tiempo lo que suele provocar la aparición de resistencias que serán contrarrestadas, finalmente, por la sustitución de la mano de obra. En la primavera de 2018, las movilizaciones colectivas de temporeras marroquíes contratadas en origen pusieron en cuestión, por primera vezy públicamente, este modelo de contratación. Al final de la temporada, varios agentes informaron de que un número importante de trabajadores no había regresado a Marruecos. Entendemos estas formas de resistencia colectivas e individuales, la voz y la salida (Hirschman, 1970), las manifestaciones y las fugas, como signos de erosión de este programa tras dos décadas de funcionamiento. 
Estos hechos nos recuerdan que, entre los muchos impases generados por el actual sistema agroalimentario, cuyo impacto medioambiental o efectos negativos sobre la salud de los consumidores empiezan a ser reconocidos, la violencia ejercida sobre las trabajadoras y trabajadores es una consecuencia directa de nuestra forma de alimentarnos. En la región mediterránea, decenas de miles de jornaleros migrantes trabajan en condiciones muy precarias en los enclaves de agricultura intensiva. Privada de la mayoría de los derechos fundamentales, esta mano de obra es un pilar fundamental de estas producciones agrícolas globalizadas, especialmente lucrativas. Estas violencias, afectan especialmente a las mujeres extranjeras para las que, la insostenibilidad ecológica y social del modelo, tiene lugar en sus propios cuerpos. Hemos visto que la explotación de esta fuerza de trabajo resulta posible gracias a un régimen migratorio que legaliza y subvenciona un sistema de gestión laboral basado en la discriminación de género, que vulnera simultáneamente los derechos de las mujeres, los extranjeros y los trabajadores. La protección de los derechos de estas trabajadoras requiere la deconstrucción previa de dos paradigmas. En primer lugar, el paradigma de gestión global de las migraciones, promovido por las instituciones internacionales y la Unión Europea, que defiende la idea de que la migración temporal es beneficiosa para todos los actores pues permite proteger a los trabajadores y trabajadoras y contribuir al desarrollo de los países de origen (Geiger y Pécoud, 2013). En segundo lugar, es necesario desnaturalizar las categorizaciones racistas y sexistas que hacen recaer la responsabilidad de las violencias existentes en los campos agrícolas sobre el patriarcado marroquí, al que se acusa de producir agresores sexuales $y$ traficantes y mujeres o sumisas o prostitutas.

\section{Referencias}

Arizpe, L. y Aranda, J. (198I). The "Comparative Advantages" of Women's Disadvantages: Women Workers in the Strawberry Export Agribusiness in Mexico. Signs, 7 (2).

Basok,T. (2002). Tortillas and Tomatoes:Transmigrant Mexican Harvesters in Canada. McGill-Queen's University Press.

Becerril Quintana, O. (2007). Lucha cultural por la dignidad y los derechos humanos. Transmigrantes mexicanos en Canadá contendiendo el género, la sexualidad y la identidad (Tesis Doctoral). Universidad Autónoma Metropolitana Unidad Iztapala.

Berlan, J. P. (1987). La agricultura mediterránea y el mercado de trabajo: ¿una California para Europa? Agricultura y Sociedad, 42.

Berndt, C. y Boeckler, M. (20I4). B/Ordering the Mediterranean: Free Trade, Fresh Fruits and Fluid Fixity. En Gertel, J. y Sippel, S. (Eds.). The social cost of eating fresh (pp. 23-34). Routledge.

Biemann, U. y Sanders, A. (2003). Europlex.Video Essay.

Burawoy, M. (1976). The Functions and Reproduction of Migrant Labor: Comparative Material from Southern Africa and the U.S. American Journal of Sociology, 5, 1050-1087.

Carrasco Bengoa, C. (2006). La economía feminista: una apuesta por otra economía. En Vara, M.J. (Ed.). Estudios sobre Género y Economía (pp. 29-62). Akal.

Delgado Cabeza, M.y Aragón Mejías, M.A. (2006). Los campos andaluces en la globalización:Almería y Huelva, fábricas de hortalizas. En La agricultura española en la era de la globalización (pp. 425-474). Ministerio de Agricultura, Pesca y de la Alimentación de España.

Delphy, C. (1998 [1970]). L'Ennemi principal. Economie politique du patriarcat. Editions Syllepse.

Dunezat, X. (2016). La sociologie des rapports sociaux de sexe : une lecture féministe et matérialiste des rapports hommes/femmes. Cahiers du Genre, 3.

Duntze, N. (2008). De la saison à l'univers concentrationnaire. Plein droit, 78 (3), 6-8.

Encalada Grez, E. y Preibisch, K. L. (2010). The Other Side of el Otro Lado: Mexican Migrant Women and Labor Flexibility in Canadian Agriculture. Signs, 35 (2).

Echevarría, P. (I3.10.2019). Tiempo de matar fresas: radiografía de argumentos y pruebas que la judicatura onubense no trata. La mar de Onuba.

Falquet, J. (2009). La règle du jeu. Repenser la co-formation des rapports sociaux de sexe, de classe et de "race" dans la mondialisation néolibérale. En Dorlin, E. (Ed.). Sexe, race, classe : pour une épistémologie de la domination (pp. 177-195). PUF.

Federici, S. (2014). Caliban et la sorcière. Femmes, corps et accumulation primitive. Entremonde/Sansevero. 
Freshuelva (2018). El sector de los Berries de Huelva. Presente y futuro. Recuperado de: https://www.huelvainformacion. es/2018/I0/I8/DEF_charla_coloquio_Rafael_Dominguez_I8-I0.pdf?hash=db575e233334b7e35b32619e7f5 ab98180ee8Ia3 (19.04.202I).

Geiger, M. y Pécoud,A. (20I3). Disciplining the transnational mobility of people. Palgrave McMillan.

Gertel, J. y Sippel, S. R. (20I4). Seasonal workers in Mediterranean agriculture: the social costs of eating fresh. Routledge.

Gualda, E. y Ruiz García, M. (2004). Migración femenina de Europa del este y mercado de trabajo agrícola en la provincia de Huelva, España. Migraciones Internacionales, 4 (2), 36-65.

Guénif Souilamas, N. y Macé, É. (2004). Les féministes et le garçon arabe. Editions de l'Aube.

Guillaumin, C. (1992). Sexe, race et pratique du pouvoir: l'idée de Nature. Côté-femmes.

Harvey, D. (1989). The Condition of Postmodernity:An Enquiry into the Origins of Cultural Change. Blackwell.

Hellio, E. (20I4). Importer des femmes pour exporter des fraises? Le cas des saisonnières marocaines en Andalousie. (Tesis doctoral). Urmis, Université de Nice Sophia Antipolis.

Hellio, E. y Moreno Nieto, J. (2017). Contrataciones en origen, deslocalización productiva y feminización del trabajo en la fresicultura del norte de Marruecos y el sur de España. Una historia en común. Navegar, 5 (3), 21 -46.

Hirschman, A. O. (1970). Exit, voice, and loyalty: responses to decline in firms, organizations, and states. Harvard University Press.

Junta de Andalucía (2017). Plan estratégico provincial de los berries. Instituto de Investigación y Formación Agraria y Pesquera. Consejería de Agricultura, Agricultura Pesca y Desarrollo Rural.

Kergoat, D. (1982). Les ouvrières. Le Sycomore.

Mies, M. (1986). Patriarchy and Accumulation on a World Scale. Zed Books.

Molinero, Y. y Avallone, G. (2016). Producing Cheap Food and Labour: Migrations and Agriculture in the Capitalistic World-Ecology. Social Change Review, 2, I 2 | - | 48.

Moreno Nieto, J. (2009). Los contratos en origen de temporada: mujeres marroquíes en la agricultura onubense. REIM, 7, 58-78.

Morice, A. (2004). Le travail sans le travailleur. Plein droit, 6 I (2), 2-7.

Moore, J.W. (2015). Capitalism in the Web of Life: Ecology and the Accumulation of Capital.Verso.

Moore, J.W. (2017). The Value of Everything? Work, Capital, and Historical Nature in the Capitalist World-Ecology. Review:A Journal of the Fernand Braudel Center, 37 (3-4), 245-292.

Moore, J.W. (2017). ¿Trabajo Barato?: Tiempo, Capital y la Reproducción de la Naturaleza Humana. Relaciones Internacionales, 36, 215-232.

Moulier Boutang, Y. (1998). De l'esclavage au salariat: économie historique du salariat bridé. Presses Universitaires de France.

Müller, P. y Prandi, S. ( 18.05 .20 I8).Violadas en los Campos de Europa. BuzzFeed.News.

Reigada Olaizola, A. (2009). Las nuevas temporeras de la fresa en Huelva: flexibilidad productiva, contratación en origen y feminización del trabajo en una agricultura globalizada (Tesis Doctoral). Universidad de Sevilla.

Reigada, A., Moreno, J. y Mozo, C. (202I). Innovación y distinción bajo el objetivo de la calidad en la producción de frutos rojos en Huelva. Revista Española de Sociología, 30 (I), a I 7.

Salzinger, L. (2003). Genders in production: making workers in Mexico's global factories. University of California Press.

Sayad,A. (1986). Coûts et «profits» de l'immigration. Actes de la recherche en sciences sociales, 6I, 79-82.

Sayad, A. (199I). L'immigration ou les paradoxes de l'altérité. I, L'illusion du provisoire. De Boeck université.

Stoler, A. L. (20I3). La chair de l'empire: savoirs intimes et pouvoirs raciaux en régime colonial. La Découverte.

Tabet, P. (2004). La grande arnaque: sexualité des femmes et échange économico-sexuel. L'Harmattan.

Terray, E. (1999). Le travail des étrangers en situation irrégulière ou la délocalisation sur place. En Balibar, E., Chemillier-Gendreau, M., Costa-Lascoux, J. y Terray, E. Sans-papiers: l'archaïsme fatal (pp. 9-34). La découverte.

Varios autores (2006).wl@s trabajadores de la fresa en Huelva. Editorial Atrapasueños.

Walker, R. y Moore, J.W. (20I8).Value, nature, and the vortex of accumulation. En Ernston, H. y Swyngedouw, E. (Eds.). Urban political ecology in the anthropo-Obscene. Routledge.

Zeneidi, D. (20 I3). L’enfermement à la campagne? Hommes \& Migrations, I30I (I), 9-I6. 


\section{RELACIONES INTERNACIONALES}

Revista académica cuatrimestral de publicación electrónica Grupo de Estudios de Relaciones Internacionales (GERI)

Universidad Autónoma de Madrid, España

https://revistas.uam.es/relacionesinternacionales

ISSN 1699 - 3950

f facebook.com/RelacionesInternacionales

3. twitter.com/RRInternacional 